\title{
Characterization of Pt-Si interface by spectroscopic ellipsometry
}

\author{
Y. C. Liu, T. P. Chen, S. Fung, and C. D. Beling \\ Department of Physics, University of Hong Kong, Hong Kong
}

(Received 25 May 1994; accepted for publication 23 August 1994)

\begin{abstract}
Spectroscopic ellipsometric measurements for $\mathrm{Pt} / n$-Si samples with different thickness of $\mathrm{Pt}$ films have been performed. The thickness of the Pt films determined with the three-phase model (air/Pt/ $\mathrm{Si})$ changes with the wavelength $\lambda$ while that with the four-phase model (air/Pt/interface layer/Si) remains unchanged, showing the existence of an interface layer. At the same time, the apparent optical dielectric constants of the interface layer as a function of $\lambda$ are also obtained. A calculation based on the effective medium theory is carried out to simulate the optical dielectric data of the interface layer. Some structural information of the interface layer is obtained from the calculation. () 1994 American Institute of Physics.
\end{abstract}

\section{INTRODUCTION}

The electronic properties of metal-semiconductor interfaces offer considerable evidence of their dependence on the structural and chemical features of the interfaces. ${ }^{1,2}$ Any physical and chemical changes occurring at the interfaces may influence the properties of the Schottky barriers. There have been a great deal of efforts attempting to correlate the chemical and structural properties of the interfaces with the observed Schottky barrier heights. ${ }^{1}$ The developments in surface sensitive techniques such as photoemission spectroscopy (PES), secondary-ion mass spectroscopy (SIMS), Auger electron spectroscopy (AES), etc., have led to a more complete understanding of the properties. However, it is still difficult to investigate nondestructively a narrow interface region with a width of between several $\AA$ and several ten $\AA$ by these techniques, because of the problems of the techniques' depth resolution or of the influence of the combined ion etching.

Spectroscopic ellipsometry (SE) is a sensitive nondestructive optical technique. ${ }^{3}$ In this work, we use this technique to investigate metal-semiconductor interfaces. As an example, the interface of platinum on chemically clean silicon surface is studied. For some metals such as Pd and Pt, the atom intermixing and chemical bonding may occur at the metal-Si interfaces at room temperature. ${ }^{4-6}$ In addition, for a chemically clean Si surface there is a native oxide layer on the Si surface. Therefore, an interface layer whose physical and chemical properties differ from those of a pure metal film and the Si substrate may exist at the Pt-Si interface. The present study clearly shows the existence of such an interface layer. Meanwhile, the apparent optical dielectric constants of the interface layer as a function of the wavelength are also obtained in this study. A calculation based on the effectivemedium theory (EMT) is carried out to simulate the constants, and thus some useful information of the interface structure is obtained.

\section{EXPERIMENTAL}

The substrates used in this study are n-type silicon wafers of (111) orientation with a doping concentration of $5 \times 10^{15} \mathrm{~cm}^{-3}$. The wafers are cleaned with ultrasonic treatment sequentially in acetone, absolute methanol, and de- ionized water $(20 \mathrm{M} \Omega \mathrm{cm})$. The wafers are then first put into hot $\mathrm{H}_{2} \mathrm{SO}_{4}: \mathrm{H}_{2} \mathrm{O}_{4}: \mathrm{H}_{2} \mathrm{O}(10: 1: 1)$ solution for $10 \mathrm{~min}$ and then rinsed in de-ionized water. Afterward, without drying, the wafers are etched in $\mathrm{HF}: \mathrm{HNO}_{3}: \mathrm{H}_{2} \mathrm{O}(3: 1: 1)$ solution for another $1 \mathrm{~min}$, and finally, they are rinsed in de-ionized water. The platinum thin films are evaporated onto the surfaces of the Si wafers by an electron-beam evaporator in a vacuum of $\sim 10^{-6}$ Torr. In this study, a number of samples with different $P t$ thickness are fabricated under identical conditions, and the thickness of the Pt films is in the range of $30-120 \AA$. . The ellipsometric measurements which yield the values of the parameters of $\Psi$ and $\Delta$ at each wavelength are carried out with a spectral ellipsometer of the rotating analyzer type $\mathrm{e}^{8-10}$ in the wavelength range of $3500-6500 \AA$. The measurements are performed at room temperature in air. The light source is a $75 \mathrm{~W}$ Xe lamp, and a monochromatic beam is provided by a grating monochromator with a grating of 1200 lines $/ \mathrm{mm}$. All the spectra are taken at an angle of incidence of $70^{\circ}$.

\section{DETERMINATION OF OPTICAL RESPONSE OF Pt-Si INTERFACE BY ELLIPSOMETRY}

\section{A. Theory and method}

An ellipsometric measurement determines the phase and amplitude of the complex reflectance ratio

$$
\rho=R_{p} / R_{s}=\operatorname{tg} \Psi \exp (i \Delta),
$$

where $R_{s}$ and $R_{p}$ are the complex field reflectance for $s$ - and $p$ - polarized light (perpendicular to and parallel with the plane of incidence), respectively, and $\operatorname{tg} \Psi$ and $\Delta$ are the standard ellipsometric amplitude and phase parameters., For a four-phase system such as the system of air/Pt film/ interface layer/Si substrate as shown in Fig. 1(a), $R_{s}$ and $R_{p}$ can be expressed as

$R_{\alpha}=\left(r_{01}^{\alpha}+R_{12}^{\alpha} X_{1}\right) /\left(1+r_{01}^{\alpha} R_{12}^{\alpha} X_{1}\right) \quad(\alpha=p, s)$,

where

$X_{1}=\exp \left(-i \frac{4 \pi}{\lambda} N_{1} d_{1} \cos \phi_{1}\right)$,
$R_{12}^{\alpha}=\left(r_{12}^{\alpha}+r_{23}^{\alpha} X_{2}\right) /\left(1+r_{12}^{\alpha} r_{23}^{\alpha} X_{2}\right)$, 


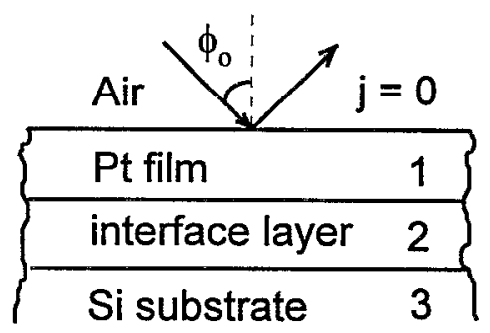

(a)

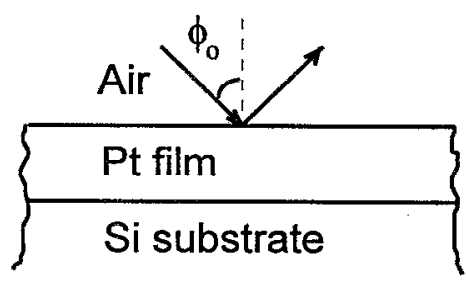

(b)

FIG. 1. Proposed models for the Pt/Si system: (a) four-phase model, and (b) three-phase model.

$X_{2}=\exp \left(-i \frac{4 \pi}{\lambda} N_{2} d_{2} \cos \phi_{2}\right)$.

The four angles $\phi_{0}, \phi_{1}, \phi_{2}$, and $\phi_{3}$ between the directions of propagation of the plane waves in phase 0 (air), 1 (Pt film), 2 (interface layer), and 3 (Si substrate), respectively, and the normal to the phase boundaries are related by Snell's law

$$
N_{0} \sin \phi_{0}=N_{1} \sin \phi_{1}=N_{2} \sin \phi_{2}=N_{3} \sin \phi_{3} \text {. }
$$

In the above equations, $\lambda$ is the free-space wavelength of light; $N_{j}=n_{j}-i k_{j}(j=0,1,2,3)$ is the complex refractive index of the $j$ th phase [see Fig. 1(a)] where $n$ is the refractive index and $k$ is the extinction coefficient (note that in the present study the refractive index and extinction coefficient of air are taken as $n_{0}=1$ and $\left.k_{0}=0\right) ; r_{j l}^{\alpha}(j, l=0,1,2,3$, and $j<l)$ is the Fresnel complex-amplitude reflection coefficient for $\alpha-(\alpha=p, s)$ polarized light at the interface between the $j$ th phase and the $l$ th phase ${ }^{7} ; d_{1}$ is the thickness of the Pt film and $d_{2}$ is the thickness of the interface layer. If $d_{2}=0$, then the four-phase system is reduced to a three-phase system as shown in Fig. 1(b) with $R_{12}^{\alpha}=r_{13}^{\alpha}(\alpha=p, s)$.

For the four-phase system, the functional dependence of $\Psi$ and $\Delta$ on the system parameters can be symbolically written as

$$
\begin{aligned}
& \Psi=f_{1}\left(n_{1}, k_{1}, n_{2}, k_{2}, n_{3}, k_{3}, d_{1}, d_{2}, \lambda\right), \\
& \Delta=f_{2}\left(n_{1}, k_{1}, n_{2}, k_{2}, n_{3}, k_{3}, d_{1}, d_{2}, \lambda\right) .
\end{aligned}
$$

Equations (7) and (8) are two real equations. The functions of $f_{1}$ and $f_{2}$ are determined with Eq. (1) together with Eqs. (2) $-(6) . f_{1}$ and $f_{2}$ cannot be expressed as analytical formulae, they can be handled only by numerical computation. As discussed below, it is possible to determine some of the system parameters with Eqs. (7) and (8).
As the optical constants $n_{3}$ and $k_{3}$ of the Si substrate are known (in fact, they can be found in the literature), the unknown parameters for the four-phase system shown in Fig. 1(a) include: the thickness $\left(d_{1}\right)$ and the optical constants $\left(n_{1}\right.$ and $k_{1}$ ) of the Pt film, and the thickness $\left(d_{2}\right)$ and the optical constants $\left(n_{2}, k_{2}\right)$ of the interface layer. At each wavelength, the ellipsometric measurement gives a $\Psi$ and $\Delta$ leading to two independent equations as (7) and (8). Thus the number of independent equations is less than the number of unknown parameters, and the equations cannot be solved definitely. We approach this problem by a multithickness method. Consider $M$ pieces of $\mathrm{Pt} / \mathrm{Si}$ samples whose fabrication conditions are identical. The thickness of the Pt films is different, but the difference is not too large. It is assumed that the optical constants of the Pt film as well as of the interface layer and the thickness of the interface layer in these samples are independent of the $\mathrm{Pt}$ film thickness provided the difference of the film thickness is not too large. This assumption has been checked carefully with ellipsometric measurements combined with calculations for many samples, and we find that it is reasonable. For these $M$ pieces of samples, only the thickness of the Pt films, which is marked as $d_{1}^{j}(j=1,2,3, \ldots, M)$, is different, and the ellipsometric measurements can provide $2 M$ independent equations:

$$
\begin{aligned}
\Psi^{j}= & f_{1}\left(n_{1}, k_{1}, n_{2}, k_{2}, n_{3}, k_{3}, d_{1}^{j}, d_{2}, \lambda\right), \\
& (j=1,2,3, \ldots, M) \\
\Delta^{j}= & f_{2}\left(n_{1}, k_{1}, n_{2}, k_{2}, n_{3}, k_{3}, d_{1}^{j}, d_{2}, \lambda\right) .
\end{aligned}
$$

In the above equations, there are $(5+M)$ unknown parameters. Obviously, we musi have $2 M \geqslant(5+M)$, or $M \geqslant 5$, so that all the unknowns can be determined definitely. In the present study, the ellipsometric measurements are performed for five samples with different thickness of $P t$ films.

If the $\mathrm{Pt} / \mathrm{Si}$ system is regarded as a three-phase system, then $d_{2}=0$, and the above equations are reduced to be independent of $n_{2}$ and $k_{2}$. In this situation, the unknown parameters include $n_{1}, k_{1}$, and $d_{1}^{j}$, and the number of the unknowns is $(2+M)$. Obviously, all the unknown parameters can bc determined definitely with $M=5$.

For both the three-phase and the four-phase systems, Eqs. (9) and (10) can only be solved by numerical computation. The computation usually entails the search of a set of unknown parameters under measurement such that the quantity

$$
F=\sum_{j=1}^{M}\left[\left(\psi_{m}^{j}-\psi_{c}^{j}\right)^{2}+\left(\Delta_{m}^{j}-\Delta_{c}^{j}\right)^{2}\right]
$$

is zero or a minimum. $F$ is an error function, $\Psi_{m}^{j}$ and $\Delta_{m}^{j}$ are the ellipsometric parameters of the $j$ th sample from the measurements, and $\Psi_{c}^{j}$ and $\Delta_{c}^{j}$ are the corresponding values of the two parameters computed with Eqs. (9) and (10). For the four-phase system $(5+M)$ unknowns are searched to minimize the error function $F$ while $(2+M)$ unknowns are searched for the three-phase system.

In the present study, we discovered another error funclion that promises to reduce the number of unknowns to the 
minimum. This error function is based on the fact that the optical constants can be separated from the film thickness $d_{1}^{j}$. From Eq. (3), $d_{1}^{j}$ can be determined as

$$
d_{1}^{j}=\frac{i \lambda \ln X_{1}^{j}}{4 \pi N_{1} \cos \phi_{1}} \quad(j=1,2,3, \ldots, M) .
$$

Mathematically, $d_{1}^{j}$ is a complex function of $\Psi^{j}, \Delta^{j}, n_{1}, k_{1}$, $n_{2}, k_{2}, n_{3}, k_{3}, d_{2}$, and $\lambda$, and it can be expressed as $d_{1}^{j}=\operatorname{Re}\left(d_{1}^{j}\right)+i \operatorname{Im}\left(d_{1}^{j}\right)$, where $\operatorname{Re}\left(d_{1}^{j}\right)$ and $\operatorname{Im}\left(d_{1}^{j}\right)$ are the real and imaginary parts of $d_{1}^{j}$, respectively. Physically, $d_{1}^{j}$ should be a positive real quantity which is independent of wavelength $\lambda$. In an ideal situation where there are no experimental and model errors, it should be possible to compute values for the unknown parameters that would make the imaginary part, $\operatorname{Im}\left(d_{1}^{j}\right)=0$, and the real part, $\operatorname{Re}\left(d_{1}^{j}\right)$, independent of $\lambda$. Therefore, the error function can be defined as

$$
F=\sum_{j=1}^{M}\left[\operatorname{Im}\left(d_{1}^{j}\right)\right]^{2} .
$$

This error function is independent of $d_{1}^{j}$. So the space of the search for the unknowns is reduced from $(2+M)$ dimensions to two dimensions $\left(n_{1}, k_{1}\right)$ for the three-phase system or from $(5+M)$ dimensions to five dimensions $\left(n_{1}, k_{1}, n_{2}, k_{2}, d_{2}\right)$ for the four-phase system. This procedure makes the computation for the unknowns much simpler. Substituting the optical constants so obtained into Eq. (12) and computing the real part $\operatorname{Re}\left(d_{1}^{j}\right)$, we obtain the thickness of the Pt films.

The validity of the models for the $\mathrm{Pt} / \mathrm{Si}$ system can be examined with the following idea: if the model is perfect, the computed thickness of the $\mathrm{Pt}$ films should not change with wavelength regularly. If the four-phase model is suitable and the three-phase model is not, there is an optically effective interface layer at the Pt-Si interface; if there is no such interface layer, the results of the computations based on both the models should be identical.

\section{B. Results and discussions}

The ellipsometric measurements and computations were carried out for five Pt/Si samples with different thickness of Pt films. The results from the computation based on the fourphase model shows that for all samples, the film thickness $d_{1}^{j}$ $(j=1,2,3,4,5)$ has only a random variation (within $5 \%)$ with wavelength, and the computed thickness $d_{2}$ of the interface layer is $(30 \pm 1) \AA$ and the random variation of $d_{2}$ with wavelength is within $3 \%$. The average thickness of the Pt films of the samples No. 1-No. 5 obtained from the fourphase model are $39,46,54,95$, and $107 \AA$, respectively. However, the computation based on the three-phase model yields a very different picture. The change of the computed thickness $d_{1}^{j}$ from this model with wavelength is shown in Fig. 2. As one can see in Fig. 2, the variation of the computed values of $d_{1}^{j}$ with the wavelength $\lambda$ depends very much on the thickness of the Pt films, and the variation is also different at different wavelength. In order to describe the difference between the three-phase model and the four-phase model, we compute the quantity

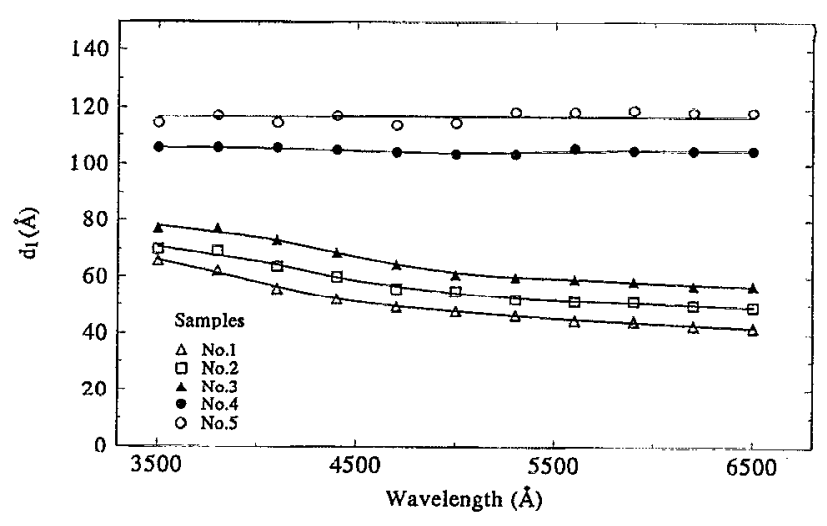

FIG. 2. Pt-film thickness $d_{1}$ determined with the three-phase model as a function of the wavelength $\lambda$.

$$
R^{j}=\frac{d_{1_{\text {three-phase }}^{j}}-d_{1}^{j}{ }_{\text {four-phase }}}{d_{1}^{j} \text { fonir-phase }} \times 100 \%,
$$

where $d_{1}^{j}{ }_{\text {three-phase }}$ and $d_{1}^{j}{ }_{\text {four-phase }}$ are the computed thickness of Pt films with the three-phase model and the four-phase model at each wavelength, respectively. It is found that $R^{j}$ depends on the thickness of the Pt films as well as on the wavelength. As the film thickness increases, $R^{j}$ decreases; $R^{j}$ also decreases with increasing wavelength. For example, at the wavelength of $3500 \AA, R^{j}$ decreases from $70 \%$ for the film thickness of $39 \AA$ to $15 \%$ for the film thickness of 107 $\AA$; on the other hand, for the film thickness of $39 \AA R^{j}$ decreases from $70 \%$ at the wavelength of $3500 \AA$ to $8 \%$ at the wavelength of $6500 \AA$. Obviously, the dependence of $R^{j}$ on the film thickness and on the wavelength is related to the influence of the interface layer. For thinner Pt film at shorter wavelength, the contribution of the optical response of the interface layer is more significant and thus the difference between the three-phase model and the four-phase model is larger.

It is evident from the above discussions that in contrast to the four-phase model, the three-phase model is not applicable to the $\mathrm{Pt} / \mathrm{Si}$ system. This indicates that the $\mathrm{Pt} / \mathrm{Si}$ system has an optically effective interface layer. The apparent optical constants $\left(n_{2}\right.$ and $\left.k_{2}\right)$ of the interface layer obtained from the computation based on the four-phase model are shown in Fig. 3, and the corresponding optical complex dielectric constant $\epsilon=\epsilon_{1}+i \epsilon_{2}\left(\epsilon_{1}=n_{2}^{2}-k_{2}^{2}\right.$ and $\left.\epsilon_{2}=2 n_{2} k_{2}\right)$ of the interface layer is shown in Fig. 4. Also the computation yields the optical constants $\left(n_{1}\right.$ and $\left.k_{1}\right)$ of the Pt film which are in good agreement with the values in Ref. 11. Comparing the optical constants of the interface layer with those of the Pt film and the $\mathrm{Si}$ substrate, we find that the interface layer is really a new phase and it can not be ignored in ellisopmetry.

\section{EFFECTIVE-MEDIUM THEORY SIMULATION TO THE COMPLEX DIELECTRIC CONSTANT OF THE INTERFACE LAYER}

As mentioned above, we have obtained the complex dielectric constant $\epsilon(\lambda)$ of the interface layer. In this section, we will show that, $\epsilon(\lambda)$ can be simulated with an effective- 


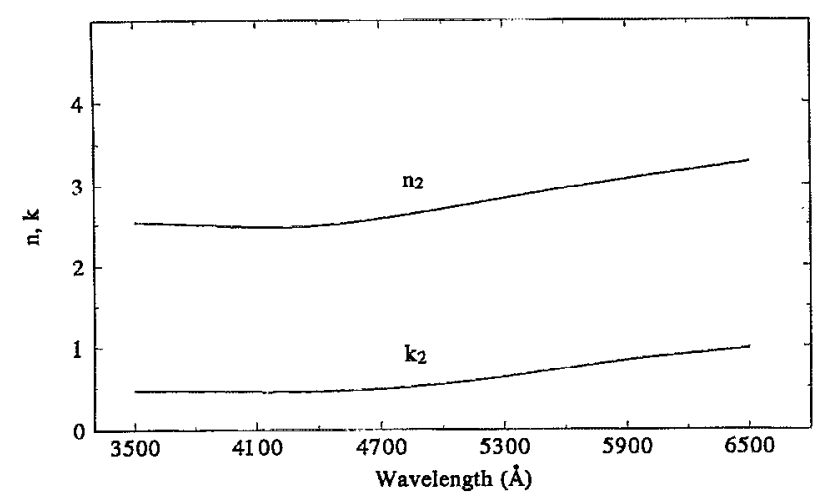

FIG. 3. Apparent optical constants $n_{2}$ and $k_{2}$ of the interface layer determined with the four-phase model.

medium theory (EMT), ${ }^{12}$ and some information of the structural properties of the interface layer can thus be obtained from such an EMT simulation.

For a chemically clean Si surface which is the situation of the present study, there is a native silicon-oxide layer $(\sim 10 \AA)$ on the surface. ${ }^{13}$ In addition, the interdiffusion of the $\mathrm{Pt}$ atoms and the $\mathrm{Si}$ atoms from the substrate may occur at the interface due to the deposition of Pt film. Therefore, it is reasonable to assume that the interface layer consists of platinum, silicon and silicon oxide. Let their complex dielectric constants be $\epsilon_{\mathrm{Pt}}, \epsilon_{\mathrm{Si}}$, and $\epsilon_{\mathrm{oxide}}$, and their fractional volumes be $Y, Z$, and $(1-Y-Z)$, respectively. The interface layer can be treated as a heterogeneous system which consists of microscopically inhomogeneous disordered materials of $\mathrm{Pt}, \mathrm{Si}$, and $\mathrm{Si}$ oxide, and its effective complex dielectric constant $\epsilon_{\text {eff }}$ can be determined from the EMT approximation:

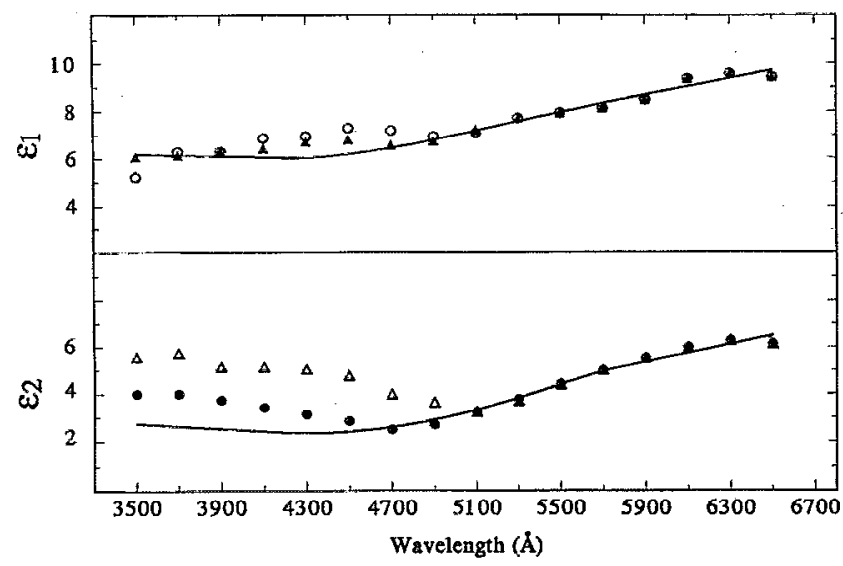

FIG. 4. Complex dielectric constant $\epsilon=\epsilon_{1}+i \epsilon_{2}$ of the interface layer as a function of the wavelength $\lambda$. The solid curves: $\epsilon_{1}$ and $\epsilon_{2}$ determined from the ellipsometric measurements with the four-phase model; the open circles and open triangles: $\epsilon_{1}$ and $\epsilon_{2}$ given by the EMT approximation with $x=2$ (i.e., $\epsilon_{\mathrm{oxide}}=\epsilon_{\mathrm{SiO}_{2}}$ ) and $Y=0.11$ and $Z=0.38$; and the full triangles and full circles: $\epsilon_{1}$ and $\epsilon_{2}$ given by the EMT approximation with $x=1.54$ (i.c., $\epsilon_{\text {oxide }}=\epsilon_{\mathrm{SiO}_{154}}$ ) and $Y=0.11$ and $Z=0.38$.

$$
\begin{aligned}
& Y\left(\frac{\epsilon_{\mathrm{Pt}}-\epsilon_{\mathrm{eff}}}{\epsilon_{\mathrm{Pt}}+2 \epsilon_{\mathrm{eff}}}\right)+Z\left(\frac{\epsilon_{\mathrm{Si}}-\epsilon_{\mathrm{eff}}}{\epsilon_{\mathrm{Si}}+2 \epsilon_{\mathrm{eff}}}\right)+(1-Y-Z)- \\
& \quad \times\left(\frac{\epsilon_{\mathrm{oxide}}-\epsilon_{\mathrm{eff}}}{\epsilon_{\text {oxide }}+2 \epsilon_{\mathrm{eff}}}\right)=0, \\
& \quad[0 \leqslant Y \leqslant 1, \quad 0 \leqslant Z \leqslant 1, \quad 0 \leqslant(1-Y-Z) \leqslant 1] .
\end{aligned}
$$

If the parameters of $\epsilon_{\mathrm{Pt}}, \epsilon_{\mathrm{Si}}$, and $\epsilon_{\mathrm{oxide}}$ are known, $\epsilon_{\mathrm{eff}}$ can be determined with Eq. (14) for given $(Y, Z)$. In the following fitting procedure, we search for the values of $(Y, Z)$ so that the difference between $\epsilon_{\mathrm{eff}}$ and $\epsilon$ is minimized over the whole measured wavelength range. This difference can be defined as

$$
\delta=\left[\frac{1}{N-1} \cdot \sum_{i=1}^{N}\left|\epsilon_{\mathrm{erI}}(\lambda)-\epsilon(\lambda)\right|^{2}\right]^{1 / 2},
$$

where $N$ is the number of measurements (wavelength measurements).

It is assumed that the $\mathrm{Si}$ oxide is $\mathrm{SiO}_{2}$, and its dielectric constants are taken from Ref. 14. The data of $\epsilon_{\mathrm{pt}}$ come from the result obtained in the present study as discussed in the above section. We take $\epsilon_{\mathrm{Si}}$ the dielectric constant of amorphous silicon. ${ }^{15}$ The result of the best fitting is shown in Fig. 4 by the open circles for $\epsilon_{1}$ and the open triangles for $\epsilon_{2}$. This fitting yields $Y=0.11, Z=0.38$. If we take $\epsilon_{\mathrm{Si}}$ the dielectric constant of crystalline silicon, we find that the difference $\delta$ is too large to carry out the fitting. This suggests that the Si component in the interface layer is in the form of noncrystalline clusters.

The above fitting allows $\epsilon_{\text {eff }}$ to fit $\epsilon$ quite-well in the range of longer wavelength while there is a large difference in the shorter-wavelength range. It is possible that the $\mathrm{Si}$ oxide is not $\mathrm{SiO}_{2}$ but $\mathrm{SiO}_{x}$. Thus, we try to improve the fitting using the dielectric constant of $\mathrm{SiO}_{x}$. The dielectric constant of $\mathrm{SiO}_{x}$ is given by ${ }^{16}$

$$
\epsilon_{\mathrm{SiO}_{x}}+\epsilon_{\mathrm{SiO}_{x}}\left(A \epsilon_{\mathrm{Si}}-B \epsilon_{\mathrm{SiO}_{2}}\right)-(A-B+1) \epsilon_{\mathrm{Si}} \epsilon_{\mathrm{SiO}_{2}}=0
$$

where

$$
\begin{aligned}
& A=\frac{1.583 x+0.4253(2-x)(x-2.123)(x+0.968)}{0.417 x-0.4253(2-x)(x-2.123)(x+0.968)}, \\
& B=\frac{0.417 x+0.09468(2-x)}{0.417 x-0.4253(2-x)(x-2.123)(x+0.968)} .
\end{aligned}
$$

This procedure leads to a better fitting in the shorterwavelength range while there is no obvious change in the longer-wavelength range, and the result is shown in Fig. 4 by the full triangles and full circles. The best fitting yields $x=1.54, Y=0.11$, and $Z=0.38$. This suggests that the Si oxide is not $\mathrm{SiO}_{2}$ but a Si-rich oxide $\mathrm{SiO}_{1.54}$. The formation of a Si-rich oxide is probably due to the interaction of platinum with the native $\mathrm{Si}$ oxide on the silicon surface. The $\mathrm{Si}-\mathrm{O}$ bonding can be broken by this interaction and thus some of $\mathrm{O}$ atoms are released. This process allows the interdiffusion of the $\mathrm{Pt}$ atoms and $\mathrm{Si}$ atoms to occur easily, and the combination of the released $\mathrm{O}$ atoms with the $\mathrm{Si}$ atoms 
from the substrate leads to the Si-rich oxide. Miller et al. ${ }^{6}$ also noted the interaction between metals such as $\mathrm{Pd}$ and $\mathrm{Au}$ and the native oxide on $\mathrm{Si}$ surfaces.

As can be seen in Fig. $4, \epsilon_{\text {eff }}(\lambda)$ from the above procedure is in good agreement with $\epsilon(\lambda)$ in a wide range of wavelength, although there are still some deviations at the short wavelengths. The deviations are probably due to the fact that only the physical mixing of $\mathrm{Pt}, \mathrm{Si}$, and $\mathrm{Si}$ oxide is considered in the EMT approximation which ignores any chemical influence. It is possible that the silicide-like substance due to chemical interaction of $\mathrm{Pt}$ and $\mathrm{Si}$ atoms forms at room temperature in the interface layer. However, there is no evidence that the stoichiometrical silicides have formed at the interface. ${ }^{17}$

\section{CONCLUSIONS}

Ellipsometric measurements for $\mathrm{Pt} / n$-Si samples have been carried out in a wavelength range of 3500-6500 A. A simple method has been used to characterize the optical response of Pt-Si interface. An interface layer whose optical properties are different from that of the Pt film and Si substrate is identified. The present study clearly shows that spectroscopic ellipsometry is a useful nondestructive method of characterizing metal-semiconductor interfaces. A calculation based on the effective-medium theory is carried out to simulate the optical dielectric constant of the interface layer obtained in this study. The result shows the existence of Si-rich oxide within the interface layer, and it also suggests that the interface layer is a region of the mixing of $\mathrm{Pt}, \mathrm{Si}$, and Si-rich oxide.

${ }^{1}$ L. J. Brillson, Surf. Sci. Rep. 2, 123 (1982); L. J. Brillson, in Handbook on Semiconductors (completely rev. edn.), edited by P. T. Landsberg (Elsevier, Amsterdam, 1992), Vol. 1, Chap. 7.

${ }^{2}$ E. H. Rhoderick and R. H. Williams, Metal-Semiconductor Contacts (Clarendon, Oxford, 1988).

${ }^{3}$ D. E. Aspnes, in Optical Properties of Solids: New Developments, edited by B. O. Seraphin (North Holland, Amsterdam, 1976).

${ }^{4}$ J. L. Freeouf, G. W. Rubloff, P. S. Ho, and T. S. Kuan, Phys. Rev. Lett. 43, 1836 (1979).

${ }^{5}$ G. W. Rubloff, P. S. Ho, J. L. Freeouf, and J. E. Lewis, Phys. Rev. B 23, 4183 (1981).

${ }^{6}$ J. N. Miller, S. A. Schwarz, I. Lindau, and W. E. Spicer, J. Vac. Sci. Technol. 17, 920 (1980).

${ }^{7}$ R. M. A. Azzam and N. M. Bashara, Ellipsometry and Polarized Light (North Holland, Amsterdam, 1977).

${ }^{8}$ D. E. Aspnes, Opt. Commun. 8, 222 (1973).

${ }^{9}$ D. E. Aspnes and A. A. Studna, Apl. Opt. 14, 220 (1975).

${ }^{10}$ L. Vina, S. Logothetidis, and M. Cardona, Phys. Rev. B 30, 1979 (1984).

${ }^{11}$ Iida Shuichi, The Constants in Physics (Scientific, Beijing, 1979) (in Chinese).

${ }^{12}$ I. Webman, J. Jortner, and M. H. Cohen, Phys. Rev. B 15, 5712 (1977).

${ }^{13}$ F. Lukes, Surf. Sci. 30, 91 (1972).

${ }^{14}$ A. J. Warneck and P. J. Lupresti, IBM J. Res. Develop. 17, 256 (1973).

${ }^{15}$ D. T. Pierce and W. E. Spicer, Phys. Rev. B 5, 3017 (1972).

${ }^{16} \mathrm{~K}$. Hubner, E. Rogmann, and G. Zuther, "Insulating Films on Semiconductors," Proc. Intern. Conf. on Insulating Films on Semiconductors, 1981, p. 30.

${ }^{17}$ L: Braicovich, I. Abbati, J. N. Miller, I. Lindau, S. Schwarz, P. R. Skeath, C. Y. Su, and W. E. Spicer, J. Vac. Sci. Technol. 17, 1005 (1980). 